\title{
A unification in the theory of linearization of second order nonlinear ordinary differential equations
}

\author{
V K Chandrasekar, M Senthilvelan and M Lakshmanan \\ Centre for Nonlinear Dynamics, Department of Physics, Bharathidasan University, \\ Tiruchirapalli - 620 024, India.
}

\begin{abstract}
In this letter, we introduce a new generalized linearizing transformation (GLT) for second order nonlinear ordinary differential equations (SNODEs). The well known invertible point (IPT) and non-point transformations (NPT) can be derived as sub-cases of the GLT. A wider class of nonlinear ODEs that cannot be linearized through NPT and IPT can be linearized by this GLT. We also illustrate how to construct GLTs and to identify the form of the linearizable equations and propose a procedure to derive the general solution from this GLT for the SNODEs. We demonstrate the theory with two examples which are of contemporary interest.
\end{abstract}

Linearizing nonlinear ordinary differential equations (NODEs) is still an open problem in the theory of differential equations [1, 2, 3]. If one raises the question whether a given arbitrary nonlinear ODE is linearizable or not, no definitive answer can be given in general. Three main points which need attention for further understanding of this problem are: (i) there is still no comprehensive literature available on the types of transformations that can linearize the ODEs, (ii) the general form of linearizable equation also differs from transformation to transformation and (iii) higher order ODEs posses a variety of linearizing transformations than the lower order ODEs. Due to these reasons no general treatment on linearizing transformations or linearizable equations has been formulated so far.

In this letter, we make an attempt to unify the linearizing transformations known for the case of second order nonlinear ODEs (SNODEs) and extend their scope. As far as the SNODEs are concerned it has been shown that, in general, one can linearize them through two different kinds of transformations. One is the well known invertible point transformation (IPT) and the other one is the non-point transformation (NPT). As far as the IPT is concerned it has been shown [3, 4, 5, 6, 7, 8, that the most general SNODE that can be linearized through such a transformation,

$$
X=F(x, t), \quad T=G(t, x),
$$

is of the form

$$
\ddot{x}=D(t, x) \dot{x}^{3}+C(t, x) \dot{x}^{2}+B(t, x) \dot{x}+A(t, x),
$$


where over dot denotes differentiation with respect to $t$ and the functions $A, B, C$ and $D$ should satisfy the following two equations,

$$
\begin{aligned}
& 3 D_{t t}+3 B D_{t}-3 A D_{x}+3 D B_{t}+B_{x x}-6 D A_{x}+C B_{x}-2 C C_{t}-2 C_{t x}=0, \\
& C_{t t}+6 A D_{t}-3 A C_{x}+3 D A_{t}-2 B_{t x}-3 C A_{x}+3 A_{x x}+2 B B_{x}-B C_{t}=0 .
\end{aligned}
$$

The transformation (11) converts the equation (2) into the linear 'free particle' equation,

$$
\frac{d^{2} X}{d T^{2}}=0
$$

On the other hand, it has also been shown that one can consider NPTs of the form

$$
X=\hat{F}(x, t), \quad d T=\hat{G}(t, x) d t,
$$

and linearize the given SNODE. The most general SNODE that can be linearized through the transformation (5) posseses the form [9]

$$
\ddot{x}+A_{2}(t, x) \dot{x}^{2}+A_{1}(t, x) \dot{x}+A_{0}(t, x)=0 .
$$

The set of relations between the functions $A_{i}$ 's, $i=0,1,2$, and the transformation (5) is given by

$$
\begin{aligned}
& A_{2}=\left(\hat{G} \hat{F}_{x x}-\hat{F}_{x} \hat{G}_{x}\right) / K, \\
& A_{1}=\left(2 \hat{G} \hat{F}_{x t}-\hat{F}_{x} \hat{G}_{t}-\hat{F}_{t} \hat{G}_{x}\right) / K, \\
& A_{0}=\left(\hat{G} \hat{F}_{t t}-\hat{F}_{t} \hat{G}_{t}\right) / K
\end{aligned}
$$

with $K=\hat{F}_{x} \hat{G} \neq 0$. The NPT also transforms equation (6) to the free particle equation (4). The functions $A_{i}$ 's, $i=0,1,2$, should satisfy the following relations [9]

$$
\begin{aligned}
& S_{1}(x, t)=A_{1 x}-2 A_{2 t}=0, \\
& S_{2}(x, t)=2 A_{0 x x}-2 A_{1 t x}+2 A_{0} A_{2 x}-A_{1 x} A_{1}+2 A_{0 x} A_{2}+2 A_{2 t t}=0 .
\end{aligned}
$$

(ii) If $S_{1}(x, t) \neq 0$ and $S_{2}(x, t) \neq 0$, then

$$
\begin{aligned}
& S_{2}^{2}+2 S_{1 t} S_{2}-2 S_{1}^{2} A_{1 t}+4 S_{1}^{2} A_{0 x}+4 S_{1}^{2} A_{0} A_{2}-2 S_{1} S_{2 t}-S_{1}^{2} A_{1}^{2}=0 \\
& S_{1 x} S_{2}+S_{1}^{2} A_{1 x}-2 S_{1}^{2} A_{2 t}-S_{1} S_{2 x}=0 .
\end{aligned}
$$

The NPT is also called a generalized Sundman transformation, see for example Refs. [10, 11].

Even though both the IPT and NPT transform the second order nonlinear ODE to the free particle equation (4), the NPT has some disadvantages over the former. For example, in the case of IPT one can unambiguously invert the free particle solution and deduce the solution of the associated nonlinear equation, whereas in the case NPT it is not so straightforward due to the non-local nature of the independent variable.

In this work, we unearth a more general transformation,

$$
X=F(x, t), \quad d T=G(t, x, \dot{x}) d t,
$$

and show that this transformation can be utilized to linearize a wider class of SNODEs and, in particular, certain equations which cannot be linearized by the NPT and IPT. We designate this transformation as the generalized linearizing transformation (GLT). 
If the function $G$ in (12) is independent of the variable $\dot{x}$ then it becomes an NPT (vide equation (5) ). On the other hand $G$ is a perfect differentiable function then it becomes an IPT, that is, $G(t, x, \dot{x})=\frac{d}{d t} \hat{G}(t, x)$, then $d T=\frac{d \hat{G}}{d t} d t \Rightarrow T=\hat{G}(t, x)$. We stress here that (12) is a unified transformation as it includes IPT and NPT as special cases.

We demonstrate our above assertion with the case where $G$ is a polynomial function in $\dot{x}$ and in particular where it is linear in $\dot{x}$ with coefficients which are arbitrary functions of $t$ and $x$. Indeed, even such a simple case leads to interesting results as we see below. To be specific we focus here on the case

$$
X=F(x, t), \quad d T=\left(G_{1}(t, x) \dot{x}+G_{2}(t, x)\right) d t .
$$

Generalizations will be dealt with elsewhere.

Substituting the transformation (13) into the free particle equation (44), the most general SODE that can be linearized through the GLT (13) can be shown to be of the form

$$
\ddot{x}+A_{3}(t, x) \dot{x}^{3}+A_{2}(t, x) \dot{x}^{2}+A_{1}(t, x) \dot{x}+A_{0}(t, x)=0
$$

and the functions $A_{i}$ 's $i=0,1,2,3$, are connected to the transformation functions $F$ and $G$ through the relations

$$
\begin{aligned}
& A_{3}=\left(G_{1} F_{x x}-F_{x} G_{1 x}\right) / M, \\
& A_{2}=\left(G_{2} F_{x x}+2 G_{1} F_{x t}-F_{x} G_{2 x}-F_{t} G_{1 x}-F_{x} G_{1 t}\right) / M, \\
& A_{1}=\left(2 G_{2} F_{x t}+G_{1} F_{t t}-F_{x} G_{2 t}-F_{t} G_{2 x}-F_{t} G_{1 t}\right) / M, \\
& A_{0}=\left(G_{2} F_{t t}-F_{t} G_{2 t}\right) / M
\end{aligned}
$$

with $M=F_{x} G_{2}-F_{t} G_{1} \neq 0$.

For the given equation one has explicit forms for the functions $A_{i}$ 's. Now solving equation (15) with the known $A_{i}$ 's one can get the linearizing transformation functions $F$ and $G$. Once $F$ and $G$ are known then using (13) we can transform (14) to the free particle equation (4) and solving the latter one can get the first integral. However, it is difficult to integrate it further unambiguously to obtain the general solution due to the non-local nature of the transformation (13). We are able to overcome this problem also here and devise a general procedure to construct the general solution. In the following we briefly describe the procedure.

Integrating the free particle equation (4) once we get

$$
\frac{d X}{d T}=I_{1}=C(t, x, \dot{x})
$$

where $I_{1}$ is the first integral. Now rewriting (16) for $\dot{x}$, we get

$$
\dot{x}=f\left(t, x, I_{1}\right),
$$

where $f$ is a function of the indicated variables. Due to non-local nature of the independent variable we need to consider only a particular solution for the free particle equation (44), that is,

$$
X(t, x)=I_{1} T
$$


from which we get

$$
x=g\left(t, T, I_{1}\right),
$$

where $g$ is a function of $t, T$ and $I_{1}$. Making use of relations (17) and (19), equation (13) can be rewritten in the form

$$
d T=h\left(t, T, I_{1}\right) d t,
$$

where again $h$ is a function of $t, T$ and $I_{1}$. We find that in the case of linearizable equations one can separate the variables $T$ and $t$ in Eq. (20) and integrate the resultant equation which in turn leads to the general solution.

In the above, we have demonstrated how to deduce linearizing transformation and the general solution for the given equation. On the other hand one can both construct linearizing transformation as well as specific linearizable equations. To illustrate this let us analyze a particular but important case of equation (14), namely, $A_{3}=0$ and $A_{2}=0$ in equation (15). Solving the first and second equation in (15) with this restriction, we obtain

$$
G_{1}=a(t) F_{x}, \quad G_{2}=a(t) F_{t}-\left(a_{t} x+b(t)\right) F_{x},
$$

where $a$ and $b$ are arbitrary functions of $t$. By using equation (21) in the last two equations in (15) we get

$$
\begin{aligned}
& A_{1}=S_{x}+\frac{a_{t}}{\left(a_{t} x+b\right)} S+\frac{\left(a_{t t} x+b_{t}\right)}{\left(a_{t} x+b\right)}, \\
& A_{0}=S_{t}+\frac{a_{t}}{\left(a_{t} x+b\right)} S^{2}+\frac{\left(a_{t t} x+b_{t}\right)}{\left(a_{t} x+b\right)} S,
\end{aligned}
$$

where

$$
S(x, t)=\frac{F_{t}}{F_{x}} .
$$

Solving equation (22) we get

$$
S=\frac{\left(c(t)-x b_{t}-\frac{1}{2} x^{2} a_{t t}+\int A_{1}\left(b+x a_{t}\right) d x\right)}{\left(b+x a_{t}\right)},
$$

where $c(t)$ is an arbitrary function of $t$. Subsituting equation (25) into (23) we obtain

$$
\begin{aligned}
A_{0}=\frac{a_{t}\left(c-x b_{t}-\frac{1}{2} x^{2} a_{t t}+\int\left(b+x a_{t}\right) A_{1} d x\right)^{2}}{\left(b+x a_{t}\right)^{3}} & \\
& +\frac{c_{t}-x b_{t t}-\frac{1}{2} x^{2} a_{t t t}+\left(\int\left(\left(b_{t}+x a_{t t}\right) A_{1}+\left(b+x a_{t}\right) A_{1 t}\right) d x\right)}{\left(b+x a_{t}\right)} .
\end{aligned}
$$

The explicit form of $F$ can be determined by substituting the expression for $S$ into (24) and solving the resultant first order partial differential equation for $F$. Once $F$ is known $G_{1}$ and $G_{2}$ can be fixed using the relation (21) which inturn provides us the GLT through (13). The associated linearizable equation assumes the form $\ddot{x}+A_{1}(x, t) \dot{x}+A_{0}(x, t)=0$, where $A_{0}$ is given in equation (26) and $A_{1}$ is the given function in this analysis. 
To illustrate the procedure with a simple but non-trivial example, let us consider the case $A_{1}=k x^{q}$, where $k$ and $q$ are arbitrary parameters, and fix the arbitrary functions $a, b$ and $c$ such as $a(t)=t, b(t)=c(t)=0$, so that the equation (25) gives us

$$
S=\frac{k}{(q+2)} x^{q+1} .
$$

Once $S$ is known $F$ and $A_{0}$ can be fixed through the relations (24) and (26) of the form

$$
A_{0}=\frac{k^{2}}{(q+2)^{2}} x^{2 q+1} \quad \text { and } \quad F=\frac{k}{q+2} t-\frac{1}{q x^{q}} .
$$

The forms of $A_{0}$ and $A_{1}$ fix the linearizable equation (14) to the form

$$
\ddot{x}+k x^{q} \dot{x}+\frac{k^{2}}{(q+2)^{2}} x^{2 q+1}=0 .
$$

Since $a(t)=t$ and $b(t)=0$, from (21) we have

$$
G_{1}=\frac{t}{x^{q+1}}, \quad G_{2}=\frac{k t}{q+2}-\frac{1}{x^{q}} .
$$

As a consequence the linearizing transformation turns out to be

$$
X=\frac{k}{q+2} t-\frac{1}{q x^{q}}, \quad d T=\left[-t\left(\frac{k}{q+2}+\frac{\dot{x}}{x^{q+1}}\right)+\frac{1}{x^{q}}\right] d t .
$$

It is easy to check that equation (29) can be linearized to the free particle equation (4) through the transformation (31).

Equation (29) and its sub-cases have been widely discussed in the contemporary literature. In particular, Mahomed and Leach [4] have shown that equation (29) with $q=1$ is one of the SNODEs that can be linearized to the free particle equation (4) through the IPT $X=\frac{k}{3} t-\frac{1}{x}$ and $T=\frac{t}{x}-\frac{k t^{2}}{6}$. Consequently, the group invariance and integrability properties of this sub-case, namely, $q=1$, and the general equation (29) have been studied extensively by different authors, see for example Refs. [12, 14, 13, 15. However, in the literature equation (29) has been shown to be linearizable to free particle equation only for the value $q=1$. For other values of $q$ the linearization of this equation through IPT or NPT was not known. But in the present work we have proved above that one can linearize the entire class of equation (29) under the one general transformation (31), irrespective of the value of $q$. One may note that choosing $q=1$ the GLT (31) coincides exactly with the point transformation for equation (29) with the same parametric restriction. This example further confirms the arguments that IPT is a sub-case of GLT.

In the following we derive the general solution of (29) using our procedure discussed through the equations (16)-(201). Using (31) in equation (16)), we obtain the first integral in the form

$$
I_{1}=\frac{\left(\frac{k}{q+2} x^{q+1}+\dot{x}\right)}{-t\left(\frac{k}{q+2} x^{q+1}+\dot{x}\right)+x} .
$$

Rewriting (32) for $\dot{x}$, we get

$$
\dot{x}=-\frac{k}{q+2} x^{q+1}+\frac{I_{1}}{\left(1+I_{1} t\right)} x .
$$


Making use of the particular solution for the free particle equation given in equation (18) and rewriting this for $x$ in equation (31), we get

$$
x=\left(\frac{1}{q\left(\frac{k}{q+2} t-I_{1} T\right)}\right)^{\frac{1}{q}} .
$$

Substituting (33) and (34) in the second equation in (31), we obtain

$$
d T=q\left(\frac{1}{1+I_{1} t}\right)\left(\frac{k}{q+2} t-I_{1} T\right) d t .
$$

Rewriting equation (35) in the form

$$
\frac{d T}{d t}+\frac{q I_{1}}{1+I_{1} t} T=\frac{k q}{q+2}\left(\frac{t}{1+I_{1} t}\right)
$$

and integrating the resultant equation, (36), we get

$$
T=\left(1+I_{1} t\right)^{-q}\left(I_{2}+\frac{\left(1+I_{1} t\right)^{q}\left(q I_{1} t-1\right)}{I_{1}^{2}\left(2+3 q+q^{2}\right)}\right)
$$

where $I_{2}$ is the second integration constant. Substituting the resultant expression for $T$ into (34) we obtain the general solution of (29), that is,

$$
x(t)=\left(\frac{I_{1}(q+1)(q+2)\left(1+I_{1} t\right)^{q}}{q\left(k\left(1+I_{1} t\right)^{q+1}-I_{1}^{2} I_{2}\left(2+3 q+q^{2}\right)\right)}\right)^{\frac{1}{q}},
$$

which is the same as the one obtained by Feix et al. [14.

Equation (29) is not an isolated example that can be linearized through the GLT. In fact, one can linearize a larger class of equations through this GLT and obtain the general solution. This is mainly due to the presence of arbitrary functions, namely, $a(t), b(t)$ and $c(t)$ in the determining equations for given $A_{1}(x, t)$. To demounstrate this, we consider a slightly more general form

$$
A_{1}=k_{1} x^{q}+k_{2}, \quad A_{3}=A_{2}=0,
$$

where $k_{1}, k_{2}$ and $q$ are arbitrary constants, in equation (14). In the present example we have included an additive constant, $k_{2}$, in the function $A_{1}$ and left the other two functions $A_{2}$ and $A_{3}$ the same as before. However, this additive constant itself enlarges the class of linearizable equations considerably, as we see below.

Let us again fix the arbitrary functions $a, b$ and $c$ of the same form as in the previous example, that is, $a(t)=t, b(t)=0$ and $c(t)=0$, so that we get $S=\frac{k_{2}}{2} x+\frac{k_{1}}{q+2} x^{q+1}$. The respective linearizable equation turns out to be

$$
\ddot{x}+\left(k_{1} x^{q}+k_{2}\right) \dot{x}+\frac{k_{1}^{2}}{(q+2)^{2}} x^{2 q+1}+\frac{k_{1} k_{2}}{q+2} x^{q+1}+\frac{k_{2}^{2}}{4} x=0
$$

and the GLT becomes

$$
\begin{aligned}
& X=\frac{2 k_{1}}{q(q+1) k_{2}}-\left(\frac{1}{q x^{q}}+\frac{2 k_{1}}{q(q+1) k_{2}}\right) e^{-\frac{q}{2} k_{2} t}, \\
& d T=\left[\frac{1}{x^{q}}\left(1-\frac{k_{2}}{2} t\right)-t\left(\frac{\dot{x}}{x^{q+1}}+\frac{k_{1}}{(q+1)}\right)\right] e^{-\frac{q}{2} k_{2} t} d t .
\end{aligned}
$$


One may note that in the limit $k_{2} \rightarrow 0$ both the linearizing transformations, (41) and the linearizable equation, (40), reduce to the earlier example (vide equations (31) and (29) respectively).

The associated first integral reads

$$
I_{1}=\frac{d X}{d T}=\frac{\left(\frac{k_{2}}{2} x+\frac{k_{1}}{q+2} x^{q+1}+\dot{x}\right)}{-t\left(\frac{k_{2}}{2} x+\frac{k_{1}}{q+2} x^{q+1}+\dot{x}\right)+x} .
$$

Repeating the same steps given in the previous example one can get the general solution for the equation (40) in the form

$$
x(t)=\left(I_{1}+t\right) e^{-\frac{k_{2}}{2} t}\left(I_{2}+\frac{q k_{1}}{(q+2)} \int_{0}^{t} e^{-\frac{q k_{2}}{2} t^{\prime}}\left(I_{1}+t^{\prime}\right)^{q} d t^{\prime}\right)^{-\frac{1}{q}},
$$

where $I_{2}$ is the second integration constant.

Next we choose the arbitrary function $a(t)$ in an exponential form, namely, $a(t)=e^{\alpha t}$, where $\alpha$ is a constant, with $b(t)=c(t)=0$. In this case we get

$$
S=\left(\frac{k_{2}+\alpha}{2}\right) x+\frac{k_{1}}{q+2} x^{q+1} \text { and } \quad \alpha=\sqrt{k_{2}^{2}-4 \lambda},
$$

where $\lambda$ is an arbitrary parameter. The functions $b, c$ and $A_{1}$ give $A_{0}$ through the relation (26) which in turn gives us a new linearizable equation of the form

$$
\ddot{x}+\left(k_{1} x^{q}+k_{2}\right) \dot{x}+\frac{k_{1}^{2}}{(q+2)^{2}} x^{2 q+1}+\frac{k_{1} k_{2}}{(q+2)} x^{q+1}+\lambda x=0 .
$$

Proceeding further we obtain the GLT in the form

$$
\begin{aligned}
& X=\left(\frac{\left(\alpha-k_{2}\right)}{2 q \lambda}-\frac{(q+2)}{q k_{1} x^{q}}\right) e^{-\frac{q}{2}\left(k_{2}+\alpha\right) t}, \\
& d T=\left[\frac{(q+2)}{k_{1} x^{q+1}} \dot{x}+\frac{(q+2)}{2 k_{1} x^{q}}\left(k_{2}-\alpha\right)+1\right] e^{-\left(\frac{q}{2} k_{2}+\frac{(q-2)}{2} \alpha\right) t} d t .
\end{aligned}
$$

Now one can check that the transformations (41) and (46) transform (40) and (45) to the free particle equation (44). The first integrals for equation (46) can be constructed of the form

$$
I_{1}=\frac{d X}{d T}=e^{-\alpha t}\left(\frac{\dot{x}+\frac{\left(k_{2}+\alpha\right)}{2} x+\frac{k_{1}}{q+2} x^{q+1}}{\dot{x}+\frac{\left(k_{2}-\alpha\right)}{2} x+\frac{k_{1}}{q+2} x^{q+1}}\right),
$$

and the general solution take the form

$$
x(t)=\left(e^{\alpha t}-I_{1}\right) e^{-\frac{1}{2}\left(k_{2}+\alpha\right) t}\left(I_{2}+\frac{q k_{1}}{(q+2)} \int_{0}^{t}\left(\frac{e^{\alpha t^{\prime}}-I_{1}}{e^{\frac{1}{2}\left(k_{2}+\alpha\right) t^{\prime}}}\right)^{q} d t^{\prime}\right)^{-\frac{1}{q}},
$$

where $I_{2}$ is the second integration constant. Equations (43) and (48) can be integrated further explicitly using the standard method [17]. To our knowledge the solutions (43) and (48) are new to the literature.

We note that in the case $q=1$, the terms on the right hand side in the second equation in (46) can be written as a perfect derivative term and consequently leads us to the same IPT for the equation (45) respectively with $q=1$ obtained in Refs. [15, 16]. 
In this paper, we have introduced a new generalized linearizing transformation which can be used to linearize a class of equations that cannot be linearized by either IPT or NPT. In fact both IPT and NPT can be derived as sub-cases from the proposed GLT. Since the independent variable is in a non-local form in the GLT, we have devised an algorithm to rewrite the new variables in terms of old variables. Needless to say, this algorithm can also be used in the case of NPT also. Importantly, we have illustrated our theory with certain concrete examples which are of contemporary interest. Naturally one can also construct GLTs involving more general forms of $\dot{x}$ in (12) and identify new linearizable equations. The procedure can also be extended to higher order ODEs. The details will be discussed separately.

The work of VKC is supported by CSIR in the form of a CSIR Senior Research Fellowship. The work of MS and ML forms part of a Department of Science and Technology, Government of India sponsored research project.

\section{References}

[1] Olver P J 1995 Equivalence, Invariants, and Symmetry (Cambridge: Cambridge University Press)

[2] Ibragimov N H 1999 Elementary Lie Group Analysis and Ordinary Differential Equations (New York: John Wiley \& Sons)

[3] Steeb W H 1993 Invertible Point Transformations and Nonlinear Differential Equations (London: World Scientific)

[4] Mahomed F M and Leach P G L 1985 Quaestiones Math. 8 241; 198912121

[5] Duarte L G S, Duarte S E S and Moreira I C 1987 J. Phys. A: Math. Gen. 20 L701;1989 22 L201

[6] Duarte L G S, Euler N, Moreira I C and Steeb W H 1990 J. Phys. A: Math. Gen. 231457

[7] Duarte L G S, Moreira I C Euler N and Steeb W H 1991 Physica Scripta 43449

[8] Euler N, Steeb W H, Duarte L G S and Moreira I C 1991 Int. J. Theor. Phys. 301267

[9] Duarte L G S, Moreira C and Santos F C 1994 J. Phys. A: Math. Gen. 27 L739

[10] Euler N and Euler M 2004 J. Nonlinear Math. Phys. 11399

[11] Euler N, Wolf T, Leach P G L and Euler M 2003 Acta Appl. Math. 7689

[12] Davis H T 1962 Introduction to Nonlinear Differential and Integral Equations (New York: Dover)

[13] Leach P G L, Feix M R and Bouquet S 1988 J. Phys. A: Math. Gen. 29 2563; Leach P G L 1985 J. Math. Phys. 26 2510; Lemmer R L and Leach P G L 1993 J. Phys. A: Math. Gen. 26 5017;

[14] Feix M R, Geronimi C, Cairo L, Leach P G L, Lemmer R L and Bouquet S 1997 J. Phys. A: Math. Gen. 307437

[15] Chandrasekar V K, Senthilvelan M and Lakshmanan M 2005 Proc. R. Soc. London A461 2451

[16] Chandrasekar V K, Senthilvelan M and Lakshmanan M 2005 Chaos, Solitons and Fractals 261399

[17] Gradshteyn I S and Ryzhik I M 1980 Table of Integrals, Series and Products (London: Academic press) 